\title{
STUDIES ON PAIN : DISCRIMINATION OF DIFFERENCES IN INTENSITY OF A PAIN STIMULUS AS A BASIS OF A SCALE OF PAIN INTENSITY
}

\author{
By JAMES D. HARDY, HAROLD G. WOLFF, AND HELEN GOODELL \\ (From the Russell Sage Institute of Pathology, The New York Hospital, and the Departments \\ of Physiology, Medicine, and Psychiatry, Cornell University Medical College, \\ New York City)
}

(Received for publication July 9, 1947)

\section{INTRODUCTION}

In the century which has elapsed since Weber first pointed out that the ability to discriminate just noticeable differences in 2 stimuli inducing sensation depends upon the magnitude of the stimulus, psychologists and physiologists have made use of this relationship for the study of nearly all types of sensation (1). Pain has not been so studied, perhaps because of the view formerly held that pain was not a sensory entity but was the endpoint of over-stimulation of any of the recognized sensory mechanisms of the body (2). Recent evidence, however, makes it appear likely that pain per se is a sensation with specific sensory apparatus in the skin and deeper tissues, and with its own neural pathways and functional properties(3).

Investigation of the Weber ratio $(\Delta \mathrm{I} / \mathrm{I}$, in which $\mathrm{I}=$ intensity) has lead to at least 3 useful formulations about sensations other than pain.

1. The range of effective intensities of the adequate stimulus has been determined. For example, the range of effective stimuli for vision between threshold stimulus and the "dazzle" point is approximately 10 billion fold (4). For warmth sense, this range from threshold to onset of thermal pain is about 2 thousand fold (5).

2. The number of just noticeable differences which the average individual can distinguish in the range of effective stimuli has also been ascertained. Thus, there are approximately 570 barely distinguishable steps for vision in the range of intensity from complete darkness to the dazzle point (6) and about 90 steps between the warmth threshold and the thermal pain threshold (7).

3. It has been suggested that large changes in the numerical value of the Weber ratio are associated with changes in the quality of sensation. As an example may be cited the attempt to correlate the change in $\Delta \mathrm{I} / \mathrm{I}$ at about 0.02 millilambert with the change from achromatic to chromatic vision (8).

The Weber Law states simply that as the intensity of a stimulus increases, the ability to discriminate differences in intensity of stimulus decreases proportionately. For a stimulus of intensity $\mathrm{I}$, there is a minimum change in intensity, $\Delta \mathrm{I}$, which can just barely be detected when added to or subtracted from $I$. That is, $I \pm \Delta I$ can just be distinguished from $I$, and $\Delta I$ is the "just noticeable difference" (JND) in intensity of stimulus, or the difference limen (dl). Algebraically the Weber Law can be written: $\Delta \mathrm{I} / \mathrm{I}=$ $\mathrm{C}$, in which $\Delta \mathrm{I} / \mathrm{I}$ is known as the Weber ratio and $\mathrm{C}$ is a constant. This relationship has been shown to be valid over limited ranges of stimulus intensity for vision, hearing, and temperature sensation (7). It was Fechner who suggested that the constant in the equation could be interpreted as proportional to the minimal difference in sensation $(\Delta \mathrm{S})$ and that:

$\mathrm{C}=\mathrm{K} \Delta \mathrm{S}$, in which $\mathrm{K}$ is a proportionality factor and $\Delta \mathrm{S}$ is the just noticeable difference in sensation.

Therefore, $\Delta \mathrm{I} / \mathrm{I}=\mathrm{K} \Delta \mathrm{S}$, or upon integration, $\mathrm{S}=\mathrm{K} \log \mathrm{I} / \mathrm{I}_{\mathrm{o}}$, where $\mathrm{S}=$ intensity of sensation evoked by the stimulus of intensity $I, I_{o}=$ threshold stimulus intensity.

The importance of this conception lies mainly in the fact that it has afforded another quantitative approach to the study of sensations. It is the purpose of this communication to present the results of measurements of the Weber ratio for cutaneous pain sensation, and to suggest the usefulness of these measurements as a basis for the evaluation of the intensity of pain sensation. 

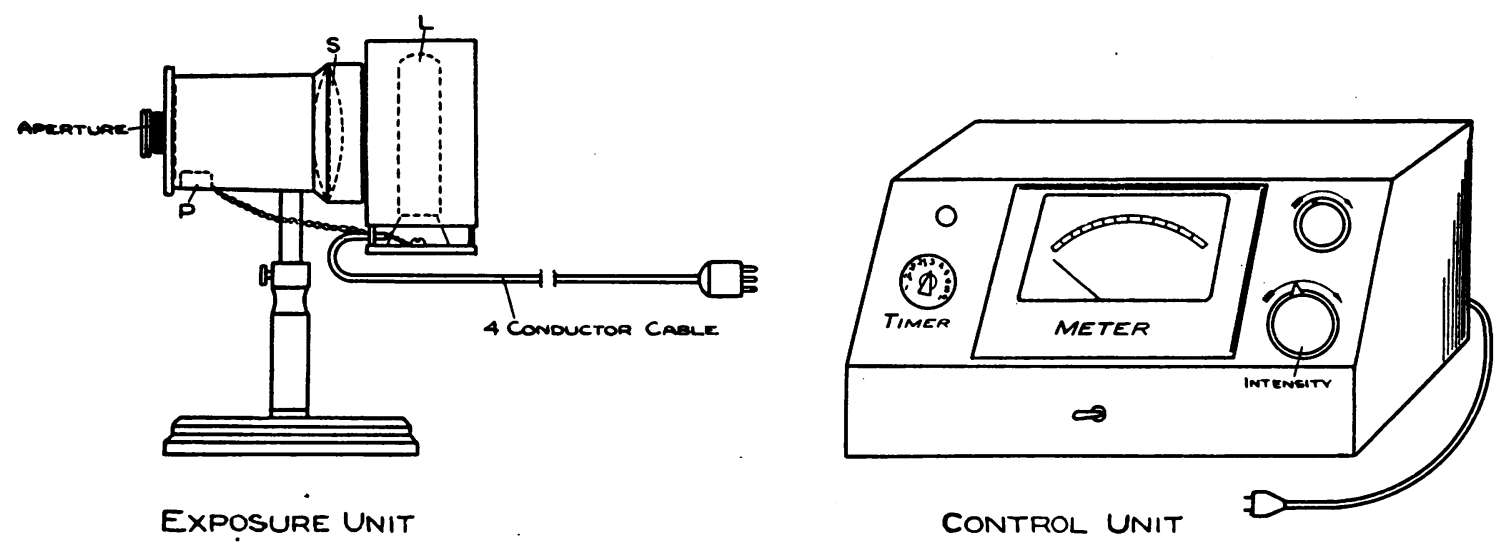

Fig. 1. Schematic Drawing of the Simplified Pain Threshold Equipment L, 500 watt lamp; S, condensing lens of $20 \mathrm{~cm}$. focus; $P$, shutter relay.

\section{METHOD}

The painful sensation which was studied in these experiments was induced in the skin by a 3-second exposure to intense heat radiation. A modified HardyWolff-Goodell pain threshold equipment was employed as the stimulator (5). The apparatus has been altered from that originally described by replacing the rheostat with a variac transformer to alter the intensity of the light beam, and the use of a sensitive voltmeter especially adapted to indicate the intensity of the radiation in millicalories per second per $\mathrm{cm}^{2}$ ( 0.001 gram cal./sec./ $\mathrm{cm}^{2}$ ). The reading of the voltmeter was checked from time to time with a calibrated radiometer. This is an essential procedure for an apparatus which depends upon the measurement of electrical input to the heat source. An electronically controlled shutter gave considerable flexibility to the equipment as it was no longer necessary to employ a pendulum to limit the time of exposure. As the changes which have been incorporated give increased flexibility and make possible a greater variety of experiments, a schematic drawing of the simplified apparatus is shown in Figure $1 .^{1}$

The method of measuring the just noticeable differences $(\Delta I)$ for the pain induced by heating the skin with radiant energy was as follows: An intensity of radiation (at or greater than the pain threshold) was selected each experimental day as the "standard" for that experiment. The method of choosing the standard was to increase systematically the intensity of the stimulus by approximately the amount of $\Delta \mathrm{I}$ as determined from the previous experiment. A series of 13 standard stimuli were used, including 220 millicalories (approximately the pain threshold). The 3 authors, serving in turn as subject and observer, were each stimulated with 2 exposures to the standard radiation. The forehead, blackened with India

1 Changes from the original design were made by the Experimental Engineering Corporation, Bergenfield, New Jersey. ink, was used as the test surface because of its uniform temperature and because this area had served satisfactorily in the past for pain threshold studies. In the series of experiments with stimuli greater than 500 millical./sec./ $\mathrm{cm}^{2}$, considerable tissue damage was produced. For this reason, a second test area, the blackened volar surface of the forearm, was chosen. This area had the same pain threshold as the forehead and was more easily cared for when blistered. Following exposure to the standard, the subjects were presented, in rotation, with 3 test stimuli. The sensation induced by the standard stimulus was compared from memory with the sensations evoked by the test stimuli and a report made as to whether the test stimuli were equal to, less than, or more than the standard. It required about 10 minutes to present the test stimuli and record the reports, as it was important not to irradiate the skin in too rapid succession. Intervals of less than 1 minute between stimulations were found to introduce uncertainty due to after-sensations. Following the first series of 3 test stimuli, the standard was again presented, the subject being so informed, and a second series of tests begun. This procedure was followed with increasing and decreasing intensities of stimulus until it became evident that $\Delta \mathrm{I}$ had been ascertained. $\Delta \mathrm{I}$ was established as the intensity difference which the subject recognized in 2 out of 3 trials.

A high degree of attention on the part of the subject was required to obtain uniform results and, in some instances, the experiment had to be delayed because of the temporary inability of a subject to concentrate sufficiently. Some improvement in discrimination was observed in the subjects as they became accustomed to the experimental procedure. This was apparent mainly in more uniform results with fewer wide variations. The subjects were agreed that this experiment required much more in the way of concentration and attention than did measurements of pain thresholds and, for this reason, conversation and interruptions during an experiment were avoided. 


\section{RESULTS}

The results of the experiment are contained in Table I. Each value of $\Delta \mathrm{I}$ reported in column 2 of Table $I$ represents an average of 3 or more observations on 3 individuals.

TABLE I

Average values of $\Delta I$ and the Weber ratio for pain sensation

\begin{tabular}{c|c|c}
\hline $\begin{array}{c}\text { Standard stimulus } \\
\text { intensities }\end{array}$ & Average $\Delta \mathrm{I}$ & Average $\frac{\Delta \mathrm{I}}{\mathrm{I}}$ \\
\hline \multicolumn{3}{|c}{ millicalories/second/cm.2 $^{2}$} \\
222 & 7 & .03 \\
240 & 7 & .03 \\
258 & 8 & .03 \\
270 & $6-$ & .02 \\
291 & 6 & .02 \\
300 & 11 & .04 \\
312 & 9 & .03 \\
330 & 15 & .05 \\
354 & 19 & .06 \\
366 & 16 & .04 \\
390 & 17 & .04 \\
420 & 29 & .07 \\
480 & 60 & .13 \\
680 & 200 & .29 \\
$(1100)$ & $(620)$ & $. .56)$ \\
\hline
\end{tabular}

In the range of stimuli from threshold to 420 millical./sec./cm. ${ }^{2}$, individual determinations of $\Delta \mathrm{I}$ usually deviated from the average value by approximately \pm 20 per cent. At the higher intensities, because of the damage done to the skin by the intense stimuli, just noticeable differences could not be so carefully ascertained, and a variation of as much as \pm 50 per cent resulted.

The relationship between the intensity of the stimulus and the "just noticeable difference" is shown in Figure 2. At threshold, $\Delta \mathrm{I}$ is approximately 7 millicalories, or \pm 3 per cent of the threshold. This value of $\Delta \mathrm{I}$ corresponds quite well with the observations previously reported on the preciseness with which the threshold can be measured by this method, that is \pm 4 per cent. There is no apparent increase in the value of $\Delta I$ between the threshold stimulus and 290 millical./ sec./cm. ${ }^{2}$, but between this latter intensity and 340 millical./sec./cm. ${ }^{2}, \Delta \mathrm{I}$ more than doubles, attaining the value of 15 millical. $/ \mathrm{sec} . / \mathrm{cm}^{2}$. Between 340 and 400 millical. $/ \mathrm{sec} . / \mathrm{cm}^{2}{ }^{2}$ there is little change in $\Delta \mathrm{I}$, but beginning at about this latter intensity, there is a final sharp rise in the $\Delta I$ values.

Thus, although 420 and 480 millical. $/ \mathrm{sec} . / \mathrm{cm}^{2}$ can be distinguished, 480 and 580 millical./sec./ $\mathrm{cm}^{2}$ cannot, and it is not until the stimulus has been increased to 680 millical. $/ \mathrm{sec} . / \mathrm{cm}^{2}$ that a barely perceptible difference can be noted. In tests on 5 subjects, 3 subjects reported 680 as more intense than 480 millical. $/ \mathrm{sec} . / \mathrm{cm} .^{2}$ and 2 subjects reported the sensations as indistinguishable. In 2 experiments, 2 subjects reported pain intensity evoked by 1100 as definitely more intense than that induced by 480 millical. $/ \mathrm{sec} . / \mathrm{cm} .^{2}$ One subject reported 680 and 1100 millical./sec./ $\mathrm{cm}^{2}$ as indistinguishable. Thus discrimination is such for stimuli greater than 680 millical./sec./ $\mathrm{cm}^{2}$ that, although it is possible to distinguish these stimuli from 480 millical. $/ \mathrm{sec} . / \mathrm{cm}^{2}$, it is not possible to make a distinction between these high stimuli themselves. The sensation evoked by a stimulus of about 680 millical./sec./cm. ${ }^{2}$ is therefore a "ceiling" pain since stimuli of greater intensity cause no perceptibly greater pain. Beyond the ceiling pain, $\Delta \mathrm{I}$ increases by the amount the intensity of the stimulus is raised above that causing the ceiling pain. For example, $\Delta I$ for ceiling pain is -200 millical./sec./cm. ${ }^{2}, \Delta \mathrm{I}$ for 1100 millical./sec./cm. ${ }^{2}$ is $-(200+420)$ millical./sec./cm. ${ }^{2}$, the latter quantity being simply the difference between 1100 millical. $/ \mathrm{sec} . / \mathrm{cm}^{2}{ }^{2}$ and the ceiling stimulus intensity. In the intensity range above 400 millical./sec./cm. ${ }^{2}, \Delta \mathrm{I}$ is changing so rapidly that the discrimination for higher intensities is considerably less than that for lower intensities. For example, 420 can be distinguished from 390 with a $\Delta \mathrm{I}$ of 30 millical./sec./cm. ${ }^{2}$ whereas 420 can barely be distinguished from 480 with a $\Delta \mathrm{I}$ of 60 millical./sec./cm. ${ }^{2}$.

\section{DISCUSSION}

\section{a. The stimulus}

It is probable that the magnitude of the $\Delta \mathrm{I}$ and its ratio to the intensity $I$ will depend to a large extent upon the type of stimulus used, and it is to be expected that measurements made with electrical and other types of stimulus will not necessarily correspond with the values reported here. It is desirable that the stimulus chosen be one for which the quantity measured is proportional to the amount of pain producing activity at the pain endings, that is, the algesic action of the stimulus. This is of importance because measurements of 


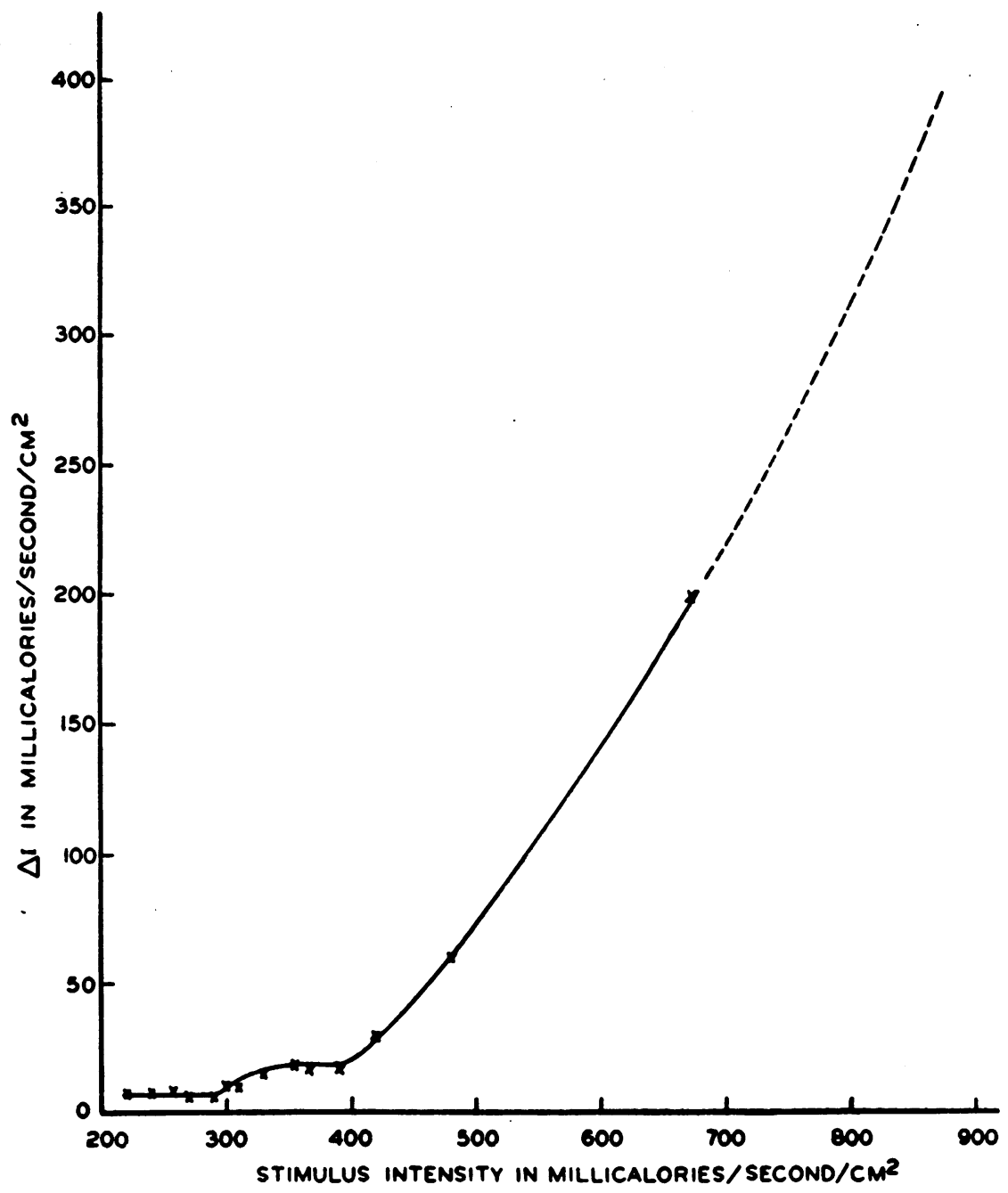

Fig. 2. $\Delta$ I for Intensities of Thermal Radiation Inducing Cutaneous Pain

$\Delta \mathrm{I}$ with an indirect type of stimulus, such as the electrical stimulation of the tooth, may require a different interpretation. For example, the direct electrical stimulation of a nerve fiber subserving pain should yield a series of $\Delta \mathrm{Is}$ which are dependent upon the condition of the nerve fiber and its electrical relation to the stimulating electrode. In the usual investigations of sensation, attention has been rightly focused on the "adequate" stimulus and, as radiant heat stimuli affect pain endings in a physiological manner, it is likely that thermal radiation is an adequate stimulus for producing pain sensation in the skin. It is apparent from Table I that by means of a suitable stimulus, reproducible measurements can be made of the just noticeable difference of painful stimuli.
The painful sensation caused by the highest stimulus intensities is distinctly different from the pain experienced in the stimulus range below 480 millical./sec./cm. ${ }^{2}$. After the greater stimuli, the subjects reported a deep aching quality as well as the bright burning quality during the 3 -second exposure. At the termination of the exposure, the deep aching pain persisted at a high intensity for some seconds as an after-sensation. For the weaker stimuli, the after-sensation had a low intensity burning quality which was generally not observed after a strong stimulus. This difference in the after-sensations may be due to the functional elimination by damage of most of the superficial endings, and to the stimulation of the deeper pain endings. The most intense stimuli 
evoke the ceiling pain in about $1 / 2$ second and the level of pain does not increase during the exposure time, whereas the weaker stimuli evoke sensations which build up during the exposure. This experience supports the idea that stimuli greater than 680 millical./sec./cm. ${ }^{2}$ evoke the "ceiling" pain.

The reproducibility in any given individual of the amount and nature of the damage caused in the skin by the thermal radiation suggests the possible usefulness of this technique in the production of experimental lesions. For example, on the skin of the volar surface of the forearm, stimuli of 400 to $480 \mathrm{millical} . / \mathrm{sec} . / \mathrm{cm}^{2}{ }^{2}$ caused erythema; those of 500 to 700 millical./sec./cm. ${ }^{2}$ caused bleb formation, whereas those of approximately 1000 millical./sec./cm. ${ }^{2}$ caused necrosis without obvious bleb formation except at the margins of the exposed area.

\section{b. The Weber ratio for pain}

The value of $\Delta \mathrm{I} / \mathrm{I}$ is approximately constant in the range of stimuli from threshold to about 320 millical./sec./cm. ${ }^{2}$, beyond which intensity there is an increase in the Weber ratio. An interpretation of Figure 2 may be made on a basis similar to that for evaluations of the Weber Ratio for stimuli producing other types of sensation. That is, the range of intensities between 220 and 320 millical./sec./cm. ${ }^{2}$ represents the stimulation of a type of cutaneous pain for which $\Delta \mathrm{I} / \mathrm{I}=$ constant $=0.03$. At about 320 millical. $/ \mathrm{sec} . / \mathrm{cm}^{2}$ another sensory element may have been added to the total sensory experience. This agrees with the experience that the quality of the pain sensation changes at about this point from a definite pricking sensation to one with an added burning quality. Indeed, this particular sensation has been chosen by 1 investigator as the pain threshold sensation rather than the sensation of minimal pricking pain which begins at about 220 millical./sec./cm. ${ }^{2}$ for a 3-second exposure (9).

\section{c. A scale of pain intensity.}

That pain has the property of intensity is well recognized. Estimates of the intensity of painful sensations have been made commonly in terms such as severe, moderate or mild, and, in the past, the judgments of the subject have been useful in experimental and clinical procedures. Although it is not possible to say that the sensation induced by a given painful stimulus in one individual will be exactly reproduced, under apparently the same experimental conditions, in another individual, on the basis of the observed predictability of behavior and of report this appears to be quite likely. Also, useful scales have been devised for other sensations, such as the scale of visual brightness and the scale of the loudness of sound (10). Therefore, on the basis of the predictability of sensory phenomena in general, we might expect to set up a sensory scale of pain and, from the data presented in Table I, we have made such an attempt.

The stimulus intensity which gives rise to the threshold sensation marks the lower limit of the range of effective stimuli. The threshold sensation is arbitrarily assigned the value 0 , meaning simply the beginning of the sensory scale. A stimulus which induces the ceiling pain determines the upper limit of the scale.

TABLE II

Scale of pain intensity

\begin{tabular}{|c|c|c|c|c|}
\hline $\begin{array}{l}\text { Stimulus } \\
\text { intensity }\end{array}$ & $\begin{array}{l}\text { Size of } \\
\text { step }\end{array}$ & $\begin{array}{c}\text { Number of } \\
\text { steps }\end{array}$ & $\begin{array}{c}\text { Number of } \\
\text { dols }\end{array}$ & $\begin{array}{l}\text { Amount of } \\
\text { stimulus } \\
\text { above } \\
\text { threshold }\end{array}$ \\
\hline \multicolumn{2}{|c|}{ millicalories/second $/ \mathrm{cm} .^{2}$} & & & $\begin{array}{l}\text { millical.| } \\
\text { sec. } / \mathrm{cm} .^{2}\end{array}$ \\
\hline \multirow{15}{*}{$\begin{array}{l}220 \\
227 \\
234 \\
241 \\
248 \\
255 \\
262 \\
269 \\
276 \\
283 \\
290 \\
300 \\
310 \\
320 \\
335 \\
350 \\
365 \\
380 \\
395 \\
425 \\
480 \\
680\end{array}$} & 7 & 1 & $\mathbf{0}$ & 0 \\
\hline & 7 & 2 & 1 & 14 \\
\hline & $\begin{array}{l}7 \\
7\end{array}$ & $\begin{array}{l}3 \\
4\end{array}$ & 2 & 28 \\
\hline & $\begin{array}{l}7 \\
7\end{array}$ & $\begin{array}{l}5 \\
6\end{array}$ & 3 & 42 \\
\hline & 7 & 7 & & \\
\hline & 7 & 8 & 4 & 56 \\
\hline & 7 & 10 & 5 & 70 \\
\hline & 10 & 11 & 6 & 00 \\
\hline & $\begin{array}{l}10 \\
10\end{array}$ & 12 & & \\
\hline & 15 & 14 & 7 & 115 \\
\hline & $\begin{array}{l}15 \\
15\end{array}$ & $\begin{array}{l}15 \\
16\end{array}$ & 8 & 145 \\
\hline & 15 & 17 & & \\
\hline & 15 & 18 & 9 & 175 \\
\hline & $\begin{array}{l}30 \\
55\end{array}$ & $\begin{array}{l}19 \\
20\end{array}$ & 10 & 265 \\
\hline & 200 & 21 & & \\
\hline
\end{tabular}

From Table II, it can be seen that there are 21 barely perceptible steps between the threshold sensation and the ceiling pain. Except under the best experimental circumstances, it is not possible to distinguish stimuli closer together than 2 steps, 


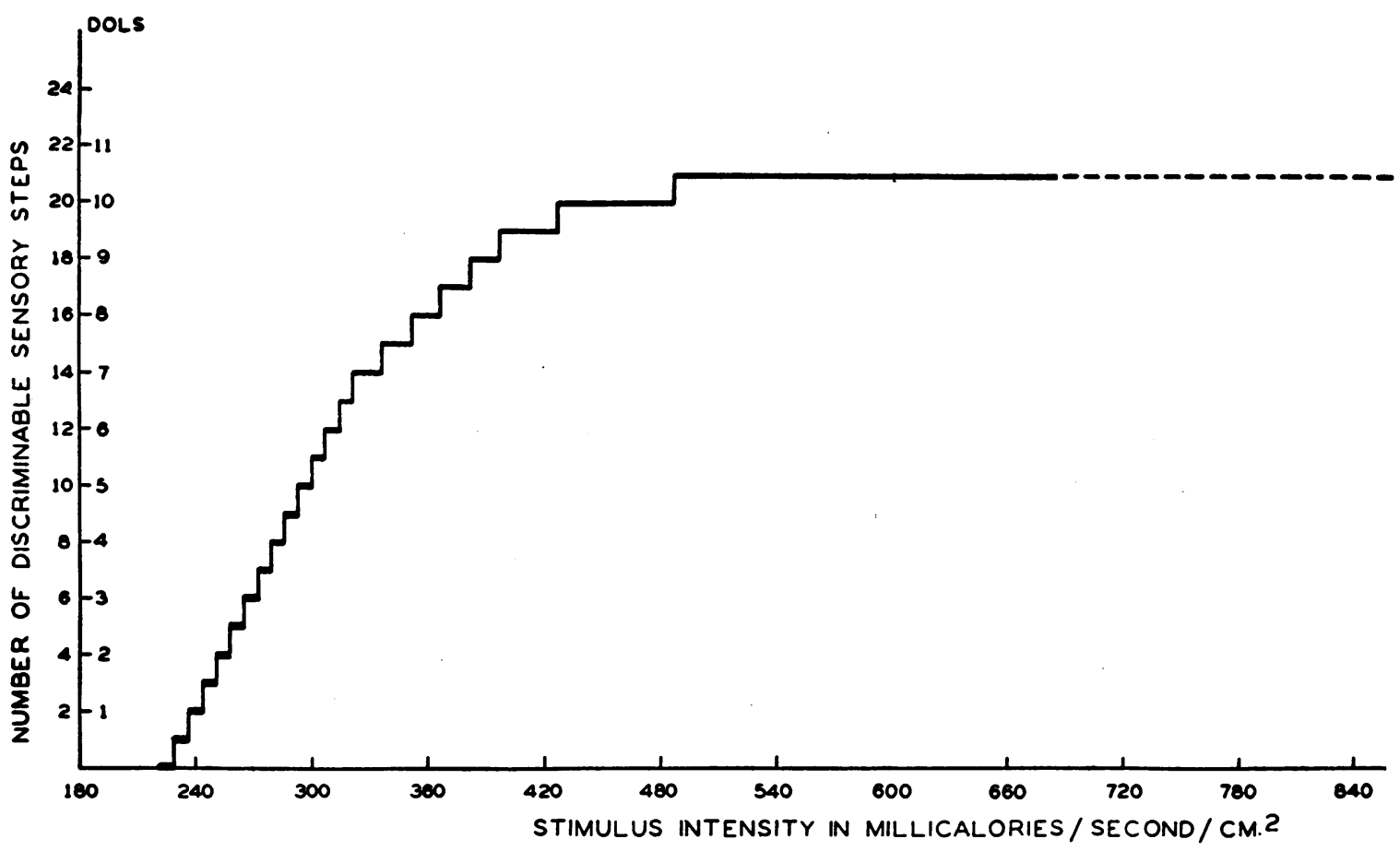

Fig. 3. Graphic Representation of Scale of Pain Intensity

and for this practical reason, the unit for pain sensation has been chosen as equivalent to 2 steps. It is suggested that the term "dol" be used to signify this unit. The ceiling pain intensity will therefore have the value of $101 / 2$ dols, as there are 21 barely discriminable steps in sensation between the pain threshold and the ceiling pain under our experimental conditions. Fortunately, as the threshold stimulus on the forehead and on the forearm is generally very near 220 millical./sec./ $\mathrm{cm}^{2}{ }^{2}$, little correction is required of the scale in Table II when these skin areas are used. A sensory scale of pain, such as that presented in Table II, is in keeping with the assumption of Fechner that the intensity of sensation evoked by a given stimulus is equivalent to the number of the discriminable steps from the threshold sensation. Figure 3 is a graphic representation of the relationship between the stimulus interisity, number of discriminable steps, and the dol scale of pain. The scale as represented in Figure 3 refers, of course, only to skin areas which have a pain threshold at 220 millical./sec./cm. ${ }^{2}$.

There are 2 advantages in setting up a scale of pain sensation on the basis of the present data, namely: (1) the sensory range is clearly defined by the threshold sensation and the maximal possible, or ceiling pain sensation, and (2) the intensity of the pain can be related to an easily controlled and reproducible adequate stimulus. The usefulness of such a scale for pain sensation is further indicated by studies which will be reported in detail elsewhere. It permits the quantitative estimation of pain intensity above the pain threshold, for instance, in the study of the nature of hyperalgesia and the effect of local and general analgesics. It also provides a useful reference scale of pain intensity against which can be made quantitative estimates of the intensities of spontaneously occurring pain in disease.

\section{SUMMARY AND CONCLUSION}

1. Measurements of just noticeable differences in estimation of painful stimuli have been made with the Hardy-Wolff-Goodell pain threshold equipment by inducing pain in the skin with thermal radiation.

2. The effective range of this stimulus is limited by the pain threshold and pain of maximal intensity. 
3. Pain induced in the skin by thermal radiation has a ceiling intensity and this ceiling pain was produced on the forearm by a stimulus intensity of 680 millical./sec./cm. ${ }^{2}$ in a 3 -second exposure.

4. The Weber ratio for pain is approximately constant between threshold and about 320 millical./ sec./cm. ${ }^{2}$. An increase in the ratio at this point suggests that an additional sensory entity with a different quality of pain has been stimulated.

5. Twenty-one discriminable intensities of pain were observed between the threshold pain and the ceiling pain.

6. On the basis of the evidence presented above, a scale of pain intensity is proposed, the unit of which is called a "dol," composed of 2 just perceptible steps in discrimination of stimulus intensity.

\section{BIBLIOGRAPHY}

1. Culler, E. A., Thermal discrimination and Weber's Law. Arch. of Psychol., 1926, 13, 81.
2. Dallenbach, K. M., Pain : History and present status. Am. J. Psychol., 1939, 52, 331.

3. Tower, S., Pain: Definition and properties of the unit for sensory reception. A. Research Nerv. and Ment. Dis., Proc., 1943, 23, 16.

4. Hecht, S., The nature of the visual process. Harvey Lectures, 1937.

5. Hardy, J. D., Wolff, H. G., and Goodell, H., Studies on pain: A new method for measuring pain threshold: Observations on spatial summation of pain. J. Clin. Invest., 1940, 19, 649.

6. Best, C. H., and Taylor, N. B., Physiological Basis of Medical Practice. Williams and Wilkins Co., Baltimore, 1943, p. 1628.

7. Herget, C. M., Granath, L. P., and Hardy, J. D., Thermal sensation and discrimination in relation to intensity of stimulus. Am. J. Physiol., 1941, 134, 645.

8. Hecht, S., Rods, cones, and the chemical basis of vision. Physiol. Rev., 1937, 17, 239.

9. Chapman, W. P., and Jones, C. M., Variations in cutaneous and visceral pain sensitivity in normal subjects. J. Clin. Invest., 1944, 23, 81.

10. Stevens, S. S., and Davis, H., Hearing. John Wiley \& Son, New York, 1938. 